\title{
DOES SUCCESSFUL BRIDGING WITH THE IMPLANTABLE LEFT VENTRICULAR ASSIST DEVICE AFFECT CARDIAC TRANSPLANTATION OUTCOME?
}

Malek G. Massad, MD

Patrick M. McCarthy, MD

Nicholas G. Smedira, MD

Daniel J. Cook, $\mathrm{PhD}^{\mathrm{b}}$

Norman B. Ratliff, $\mathrm{MD}^{\mathrm{c}}$

Marlene Goormastic, $\mathrm{MPH}^{\mathrm{a}}$

Rita L. Vargo, MS $^{\mathbf{a}}$

Jose Navia, $\mathrm{MD}^{\mathrm{a}}$

James B. Young, $\mathrm{MD}^{\mathrm{d}}$

Robert W. Stewart, MD ${ }^{\mathrm{a}}$
Objectives: We sought to determine whether cardiac transplant recipients who required a bridge to transplantation with an implantable left ventricular assist device had a different outcome than patients who underwent transplantation without such a bridge. Methods: A retrospective study of 256 cardiac transplants from 1992 to 1996 included 53 patients who received the HeartMate left ventricular assist device and 203 patients who had no left ventricular assist device support. Results: Left ventricular assist device transplants increased from $8 \%$ of all transplants in $1992(n=63)$ to $32 \%$ in $1995(n=65)$ and $43 \%$ in $1996(n=14$ year to date). Patients with and without left ventricular assist device had similar age and sex distributions. Left ventricular assist device recipients were larger (body surface area $1.96 \mathrm{vs} 1.86 \mathrm{~m}^{2}, p=\mathbf{0 . 0 0 4}$ ). They were more likely to have ischemic cardiomyopathy ( $70 \%$ vs $45 \%, p=0.001$ ) and type 0 blood group ( $51 \%$ vs $34 \%, p=0.06$ ). All patients with left ventricular assist device and $42 \%$ of those without had undergone previous cardiac operations by the time of transplantation (mean number per patient 1.5 vs $0.3, p<0.001$ ). More patients in the left ventricular assist device group had anti-HLA antibodies before transplantation (T-cell panel reactive antibody level $>10 \%$ in $66 \%$ of left ventricular assist device group vs $15 \%$ of control group, $p<0.0001$ ). Waiting time was longer for the left ventricular assist device than for patients in status I without a left ventricular assist device (median 88 vs 37 days, $p=0.002$ ). There was no difference in length of posttransplantation hospital stay (median 15 days for each) or operative mortality (3.8\% vs $4.4 \%$ ). Mean follow-up averaged 22 months. No significant difference was found in Kaplan-Meier survival estimates. One-year survival was $94 \%$ in the left ventricular assist device group and $88 \%$ in the control group (difference not significant). Comparison of posttransplantation events showed no significant difference in actuarial rates of cytomegalovirus infection $(20 \%$ vs $17 \%)$ or vascular rejection (15\% vs $12 \%)$ at 1 year of follow-up. Similar percentages of patients were free from cellular rejection at 1 year of follow-up $(12 \%$ vs $22 \%, p=0.36)$. Conclusions: Left ventricular assist device support intensified the donor shortage by including recipients who otherwise would not have survived to transplantation. Bridging affected transplant demographics, favoring patients who are larger, have ischemic cardiomyopathy, have had multiple blood
From the section of Cardiac Transplantation and Mechanical Circulatory Assist Program, Department of Thoracic and Cardiovascular Surgery, ${ }^{a}$ The Transplant Center Histocompatibility Laboratory, ${ }^{\mathrm{b}}$ The Department of Pathology, ${ }^{\mathrm{c}}$ and The Section of Heart Failure and Cardiac Transplant Medicine, Department of Cardiology, ${ }^{\mathrm{d}}$ Cleveland Clinic Foundation, Cleveland, Ohio.

Read at the Seventy-sixth Annual Meeting of The American Association for Thoracic Surgery, San Diego, Calif., April 28-May 1, 1996.
Received for publication May 6, 1996; revisions requested June 18, 1996; revisions received July 22, 1996; accepted for publication July 26, 1996.

Address for reprints: Patrick M. McCarthy, MD, Department of Thoracic and Cardiovascular Surgery, F25, Cleveland Clinic Foundation, 9500 Euclid Ave., Cleveland, OH 44195.

J Thorac Cardiovasc Surg 1996;112:1275-83

Copyright $\odot 1996$ by Mosby-Year Book, Inc.

$0022-5223 / 96 \$ 5.00+0 \quad \mathbf{1 2 / 6 / 7 6 8 2 0}$ 
transfusions and complex cardiac operations, and are HLA sensitized. Successfully bridged patients wait longer for a transplant than do UNOS status I patients without such a bridge, but they have similar posttransplantation hospital stay, operative mortality, and survival to those of patients not requiring left ventricular assist device support. (J Thorac Cardiovasc Surg 1996;112:1275-83)

$\mathrm{S}_{\mathrm{D}}^{\mathrm{t}}$ tudies from the Cardiac Transplant Research Database and the Registry of the International Society for Heart and Lung Transplantation continue to list the use of mechanical ventricular assistance as a risk factor with a significantly negative impact on survival to transplantation and on 1 year survival after cardiac transplantation., ${ }^{1,2}$ Recent studies from several centers, however, have shown comparable survivals after transplantation between patients who underwent bridge to transplantation with left ventricular assist devices (LVADs) and patients who did not have an LVAD bridge. ${ }^{3-7}$

As our experience with LVADs accrued, we noted that an increasing percentage of our patients undergoing transplantation were receiving LVAD support. It was also our impression that the subgroup of patients requiring LVAD support had an increased number of risk factors that could lead to a poor outcome. We therefore sought to determine whether cardiac transplant recipients who required a bridge to transplantation had different outcomes than did patients who did not require bridging with LVAD support.

\section{Material and methods}

Patient selection. The retrospective study comprised 256 cardiac transplant recipients at the Cleveland Clinic Foundation from January 1992 to February 1996, including 53 patients $(21 \%)$ who were bridged with the HeartMate LVAD (Thermo Cardiosystems, Inc., Woburn, Mass.) and 203 patients (79\%) who did not require bridging with LVAD support. During the period of the study, 11 additional pediatric transplants (16 years old or younger) and five adult second transplants were performed; these were excluded from the analysis. The decision to implant the LVAD was prompted by progressive deterioration of the patient's hemodynamics and organ function. The hemodynamic indications for LVAD use included patients accepted as transplant candidates who had a pulmonary capillary wedge pressure of $20 \mathrm{~mm}$ $\mathrm{Hg}$ or greater, coupled with either a cardiac index of 2.0 $\mathrm{L} / \mathrm{min}^{-1} \cdot \mathrm{m}^{2}$ or less or a systolic blood pressure of $80 \mathrm{~mm}$ $\mathrm{Hg}$ or less, despite maximal inotropic and intraaortic balloon pump support. ${ }^{6}$ Details of the device description and implantation technique have been reported elsewhere. ${ }^{8-10}$ The patients received $325 \mathrm{mg}$ aspirin once a day throughout the period of support. The pneumatic Heart-
Mate device was used in 41 LVAD-bridged transplants and the vented electric HeartMate device was used in 12. Five patients who underwent transplantation after bridging with the electric HeartMate device became outpatients during support.

Informed consent was obtained from all the patients or their families. All LVAD enrollment protocols were approved by the Cleveland Clinic Foundation Institutional Review Board. An investigational device exemption was granted by the Food and Drug Administration for use of the pneumatic HeartMate LVAD (since approved) and the vented electric HeartMate LVAD.

Transplant outcome analysis. The transplant recipients were divided into two groups: those who received an implantable LVAD as a bridge to transplantation (LVAD group) and those who did not require bridging with an implantable LVAD (Non-LVAD group). The latter group was further subdivided into two subsets of patients: those who were listed at the time of transplantation as status I on the United Network for Organ Sharing (UNOS) allocation list (status I group) and those listed as status II (status II group).

In both groups, maintenance immunosuppression consisted of a three-drug combination of cyclosporine, azathioprine, and prednisone. Patients with compromised renal function were selectively treated with OKT3 monoclonal antibody for induction, followed by conversion to cyclosporine-based immunosuppression when renal function improved. Cellular rejection was evaluated according to the grading system adopted from the criteria of Billingham and coworkers ${ }^{11}$ and the criteria of the International Society for Heart and Lung Transplantation, ${ }^{12}$ whereby International Society for Heart and Lung Transplantation grades $1 \mathrm{~A}, 1 \mathrm{~B}$, and 2 were considered mild rejection; grades $3 \mathrm{~A}$ and $3 \mathrm{~B}$ were considered moderate rejection; and grade 4 was considered severe rejection. Only patients who had moderate or severe rejection were considered in the forthcoming analysis of cellular rejection. The diagnosis of vascular rejection was based on the demonstration of immunoglobulin and complement on the coronary vascular endothelium by immunofluorescent staining, according to the criteria defined by Hammond and colleagues, ${ }^{13,14}$ in addition to evidence of endothelial cell swelling and activation on light microscopy. Immunofluorescent staining was performed on all endomyocardial biopsy specimens in the first month after transplantation and on specimens from patients who showed persistent findings of vascular rejection or hemodynamic compromise during follow-up. ${ }^{15}, 16$

Graft coronary artery disease was defined as the development of new arteriosclerosis in the cardiac allograft compared with a baseline coronary angiogram or intra- 
Table I. Annual distribution of 256 cardiac transplants at the Cleveland Clinic Foundation from January 1992 to February 1996

\begin{tabular}{lcccccr}
\hline & 1992 & 1993 & 1994 & 1995 & $1996^{*}$ & Total (1992-1996) \\
\hline LVAD implants & 8 & 15 & 13 & $32 \dot{\dagger}$ & $9 \dot{4}$ & $77 \S$ \\
LVAD transplants & 5 & 10 & 11 & 21 & 6 & 53 \\
Non-LVAD transplants & 58 & 45 & 48 & 44 & 8 & 203 \\
Total transplants & 63 & 55 & 59 & 65 & 14 & 256 \\
\hline
\end{tabular}

*January and February.

†Two patients continued on support,

$\$$ Nine patients continued on support.

§Eleven patients continued on support.

vascular ultrasonographic study done before discharge from the hospital. All patients except those who were cytomegalovirus (CMV) seronegative and who received a cardiac allograft from a CMV-seronegative donor received a 4-week course of gancyclovir prophylaxis against CMV infection. CMV hyperimmune globulin was also administered to $\mathrm{CMV}$-seronegative recipients who received an organ from a donor whose CMV serologic status was positive.

Leukocyte-depleted blood (centrifuged or filtered) was administered in most cases during support and was routinely administered after transplantation. We retrospectively defined two subsets of patients, who received less than or more than the median number of blood transfusions before transplantation, to determine the effects of blood use on the development of HLA sensitization. A T-cell panel reactive antibody (PRA) level greater than $10 \%$ was chosen to be indicative of sensitization. ${ }^{17}$ Septicemia, defined by positive blood culture results, elevated white blood cell count, and fever, combined with the need for antimicrobial treatment, ${ }^{5}$ was considered related to LVAD when the same organism was detected in the device drive line or pump. ${ }^{6}, 18$

Statistical Analysis. Comparison of demographic and clinical factors between the patients who received LVAD support and those who did not was performed and associations were analyzed with the $\chi^{2}$ test. Distributions of continuous factors were compared with the Wilcoxon rank-sum test. Kaplan-Meier survival estimates were compared with the log-rank test. Similar actuarial curves were used to compare freedoms from rejection and coronary artery disease in the two groups. A $p$ value lower than 0.05 was considered statistically significant.

\section{Results}

Demographic analysis. The donor characteristics of the LVAD and non-LVAD groups were similar. The mean donor age was 30 years (median 28 years) in the LVAD group and 31 years (median 29 years) in the non-LVAD group. There were 37 male donors for the LVAD group (70\%) and 139 male donors for the non-LVAD group $(68 \%$, not significant (NS)). Donor CMV serologic results were positive in 54\% of the LVAD group (27/53) and in $55 \%$ of the non-LVAD group $(112 / 203, \mathrm{NS})$. The average ischemic time was significantly longer in the LVAD group (157 vs 140 minutes, $p=0.007$ ).
Table I shows the annual distribution of cardiac transplants from January 1992 to February 1996. A gradual increase in the annual percentage of transplants that were bridged with LVAD support is noted. LVAD-bridged transplants increased from $8 \%$ of all transplants in $1992(n=63)$ to $32 \%$ in $1995(n=65)$ and $43 \%$ in $1996(n=14$, year to date). LVAD and non-LVAD groups had similar age and sex distributions (Table II). LVAD recipients had greater mean body weight and body surface area. Ischemic cardiomyopathy was more common in the LVAD group than in the non-LVAD group. All patients in the LVAD group and $42 \%$ in the non-LVAD group had previous cardiac operations before transplantation. The mean numbers of previous cardiac operations per patient were 1.5 and 0.3 , respectively $(p<0.001)$. Those in the LVAD group were more likely to have type O blood group $(51 \%)$ than were those in the non-LVAD group $(34 \%, p=0.06)$. All patients in the LVAD group and $62 \%$ in the non-LVAD group (126 patients) were UNOS status I at the time of transplantation (Table III). The duration of LVAD support before transplantation ranged from 3 to 153 days (median 72 days). Times on the waiting list were 88 days for the LVAD group, 37 days for non-LVAD group patients who were in UNOS status I $(p=0.002)$ and 118 days for patients in the non-LVAD group who were in UNOS status II (NS). Survival to transplantation did not differ between the LVAD and nonLVAD groups. The transplantation rate was $80 \%$ for the LVAD group on the waiting list and $84 \%$ for the non-LVAD group (NS).

Septicemia occurred in $45 \%$ of patients who had a bridge to transplantation attempted (35/77) and in $51 \%$ of patients with successful bridge to transplantation (27/53). Septicemia resulted from devicerelated infection in $42 \%$ of patients who had a bridge to transplant attempted (32/77) and in $40 \%$ of patients with successful bridge to transplantation 
Table II. Demographic Description of the 256 cardiac transplants by group, (\%)

\begin{tabular}{|c|c|c|c|}
\hline Variable & $\begin{array}{l}L V A D \\
(n=53)\end{array}$ & $\begin{array}{c}\text { Non-LVAD } \\
(n=203)\end{array}$ & $p$ \\
\hline \multicolumn{4}{|l|}{ Age (yrs) } \\
\hline Mean & 53 & 50 & NS \\
\hline Median & 53 & 53 & \\
\hline Range & $34-66$ & $17-66$ & \\
\hline \multicolumn{4}{|l|}{ Sex } \\
\hline Male $(\%)$ & $46(87)$ & $155(76)$ & NS \\
\hline Female $(\%)$ & $7(13)$ & $48(24)$ & \\
\hline Body weight (kg) & 82 & 73 & 0.0005 \\
\hline $\mathrm{BSA}\left(\mathrm{m}^{2}\right)$ & 1.96 & 1.86 & 0.004 \\
\hline \multicolumn{4}{|l|}{ Diagnosis } \\
\hline $\operatorname{ICM}(\%)$ & $37(70)$ & $91(45)$ & 0.001 \\
\hline Non-ICM $(\%)$ & $16(30)$ & $112(55)$ & \\
\hline $\begin{array}{l}\text { Previous cardiac } \\
\text { operations }(\%)\end{array}$ & $53(100)$ & $84(42)$ & 0.001 \\
\hline \multicolumn{4}{|l|}{ Blood group (\%) } \\
\hline A & $16(30)$ & $97(48)$ & \\
\hline $\mathrm{AB}$ & $2(4)$ & $14(7)$ & \\
\hline $\mathrm{B}$ & $8(15)$ & $23(11)$ & \\
\hline $\mathrm{O}$ & $27(51)$ & $69(34)$ & 0.06 \\
\hline UNOS status I (\%) & $53(100)$ & $126(62)$ & 0.001 \\
\hline $\begin{array}{r}\text { Median waiting } \\
\text { time (days) }\end{array}$ & 88 & 58 & 0.07 \\
\hline Listed UNOS status I & 73 & 37 & $<0.01$ \\
\hline $\begin{array}{l}\text { Duration of LVAD } \\
\text { support }\end{array}$ & 72 & - & \\
\hline \multicolumn{4}{|l|}{$\begin{array}{l}\text { Median blood products } \\
\text { (units)* }\end{array}$} \\
\hline On LVAD support & 96 & - & \\
\hline At transplantation & 17 & 5 & 0.0001 \\
\hline T-cell $\mathrm{PRA}>10 \%$ & $27 / 41(66 \%)$ & $25 / 169(15 \%)$ & $<0.0001$ \\
\hline CMV seropositivity (\%) & $40 / 48(83)$ & $140 / 203(69)$ & 0.04 \\
\hline
\end{tabular}

$B S A$, Body surface area; $I C M$, ischemic cardiomyopathy.

*Blood products included packed red blood cells, platelet concentrates (1 pack $=6$ units $)$, fresh-frozen plasma, and cryoprecipitate $(1$ pack $=20$ units).

(21/53). Seventy-seven percent of the patients who had septicemia during LVAD support survived to transplantation (27/35 patients), compared with $84 \%$ of the patients who did not have septicemia during support (26/31 patients, NS).

More patients in the LVAD group had anti-HLA antibodies before transplantation (T-cell PRA level $>10 \%$ in $66 \%$ of the LVAD group vs $15 \%$ of the non-LVAD group, $p<0.0001$ ). The mean peak T-cell PRA level in the LVAD group was $33 \%$, compared with $4 \%$ for the non-LVAD group $(p<$ 0.001 ). Blood use during LVAD support of more than 96 units of blood products (median) resulted in a significantly larger number of patients who were sensitized than did blood use less than the median (T-cell PRA level $>10 \%$ in $74 \%$ vs $58 \%, p<0.05$ ). A significantly higher level of sensitization against
Table III. Outcome of the 256 cardiac transplants by group, (\%)

\begin{tabular}{|c|c|c|c|}
\hline Variable & $\begin{array}{r}L V A D \\
(n=53)\end{array}$ & $\begin{array}{r}\text { Non-LVAD } \\
(n=203)\end{array}$ & $p$ \\
\hline \multicolumn{4}{|l|}{ Posttransplantation } \\
\hline Mean & 18 & 18 & NS \\
\hline Median & 15 & 15 & \\
\hline $\begin{array}{l}\text { Reexplored for } \\
\text { bleeding after } \\
\text { transplantation } \\
(\%)\end{array}$ & $3(5.7)$ & $9(4.4)$ & NS \\
\hline $\begin{array}{l}\text { 30-day operative } \\
\text { mortality }(\%)\end{array}$ & $2(3.8)$ & $9(4.4)$ & NS \\
\hline $\begin{array}{l}\text { Mean follow-up } \\
\text { (months) }\end{array}$ & 17 & 23 & 0.01 \\
\hline Survivors $(\%)$ & $48(91)$ & $174(86)$ & NS \\
\hline \multicolumn{4}{|l|}{$\begin{array}{l}\text { Posttransplantation } \\
\text { events at } 1 \mathrm{yr} \\
(\%)\end{array}$} \\
\hline $\begin{array}{l}\text { CMV infection } \\
\text { rate }\end{array}$ & 20 & 17 & NS \\
\hline $\begin{array}{l}\text { Vascular rejec- } \\
\text { tion rate }\end{array}$ & 15 & 12 & NS \\
\hline $\begin{array}{l}\text { Moderate and } \\
\text { severe rejec- } \\
\text { tion-free }\end{array}$ & 12 & 22 & NS \\
\hline $\begin{array}{l}\text { Transplant CAD } \\
\text { free }\end{array}$ & 90 & 88 & NS \\
\hline
\end{tabular}

$L O S$, Length of stay; $C A D$, coronary artery disease.

HLA antigens was also noted (mean peak T-cell PRA level $49 \%$ vs $22 \%, p=0.01$ ).

Transplant outcome analysis. Table III shows the transplant outcomes of the two groups. There was no significant difference in length of posttransplantation hospital stay between the two groups. The 30-day operative mortality rates were also similar $(3.8 \%$ vs $4.4 \%$, NS). Mean follow-up after transplantation for the two groups averaged 22 months, 17 months for the LVAD group and 23 months for the non-LVAD group $(p=0.01)$. There was a survival of $91 \%$ in the LVAD group (48/53) and a survival rate of $86 \%$ in the non-LVAD group $(174 / 203)$ at the time the study was concluded. No significant difference was found in the actuarial Kaplan-Meier survival curves between the LVAD group and the UNOS status I and II non-LVAD groups at 3 years (Fig. 1). One-year survival was $94 \%$ in the LVAD group, versus $91 \%$ and $86 \%$ in the UNOS status I and II non-LVAD groups, respectively (NS).

Comparison of posttransplantation events showed no significant difference between the LVAD and non-LVAD transplant groups with respect to inci- 


\section{PATIENT SURVIVAL AFTER TRANSPLANT BY LVAD AND UNOS STATUS}

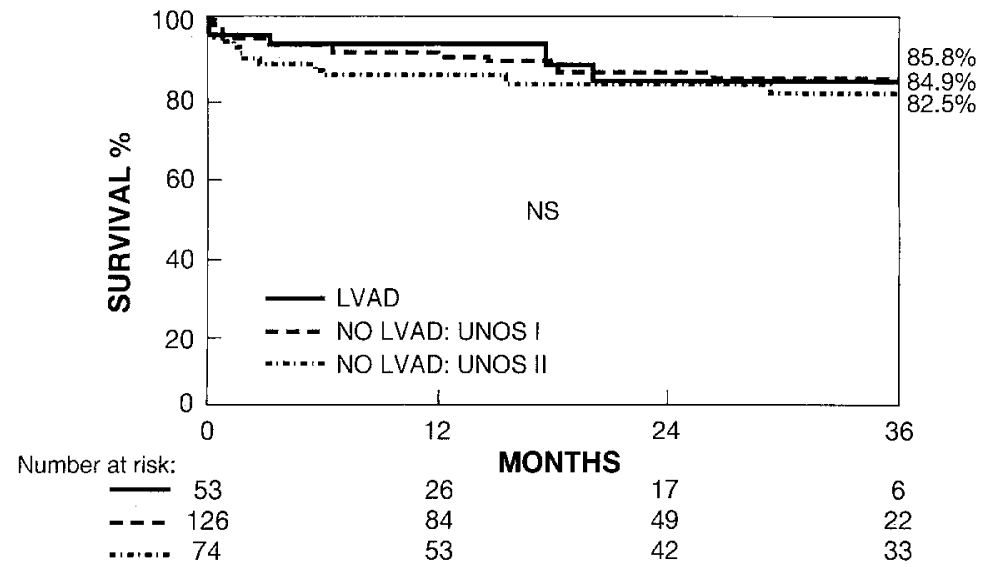

Fig. 1. Kaplan-Meier survival curves for transplant recipients with or without LVAD bridge to transplantation.

dence of CMV infection (10/53 vs 30/203 patients) and vascular rejection (7/53 vs $28 / 203$ patients) at the end of follow-up. The actuarial Kaplan-Meier estimates of the rate of CMV infection and vascular rejection in the LVAD and non-LVAD groups were not significantly different at 1 year of follow-up (Table III). Rates of freedom from transplant coronary artery disease were similar at 1 year of follow-up (90\% vs $88 \%$, NS). Rates of freedom from moderate and severe cellular rejection were also similar for the two groups (Fig. 2). The mean numbers of moderate and severe rejection episodes in the two groups at 12 weeks were 1.68 and 1.47 ; at 1 year, they were 2.53 and 1.99 episodes (NS).

\section{Discussion}

Patients who require preoperative bridging have longer waiting times, approximately $138 \%$ longer than status I patients who do not require mechanical bridging. The reason for this longer wait is multifactorial. Most of our patients with LVAD implants are inactive on the waiting list for approximately 4 weeks during which the marginal state of their vital organ function is reversed, along with their muscle wasting and malnutrition from cardiac cachexia. Furthermore, LVAD recipients are at a higher risk for sensitization against HLA antigens ${ }^{19}$ because of the large number of blood products that they receive, and they may therefore require a longer wait to identify a satisfactory match in an allograft donor. In our experience, cardiac allograft donors for LVAD recipients have similar age to donors for
non-LVAD recipients and are subjected to a longer ischemic time than are those in the latter group. This indicates that the comparable survival among LVAD transplant recipients was not a result of a preferential selection of the best local cardiac allograft donor from the UNOS allocation list; rather, it reflected these recipients' improved physical condition at the time of transplantation.

Patients who received an LVAD as a bridge to transplantation received a large number of units of blood products. Several factors contributed to the extensive perioperative blood use. Forty-nine percent of LVAD recipients had undergone previous cardiac operations by the time of LVAD implantation, and all had previous sternotomies at the time of transplantation. Data from our center indicate that hepatic dysfunction as a result of right ventricular failure or as a component of developing multiorgan dysfunction contributed to the perioperative state of coagulopathy that developed in some of these patients. Thrombocytopenia, in conjunction with heparin-associated antibodies, was observed in $40 \%$ of all patients in whom an LVAD bridge to transplantation was attempted. We maintained LVAD recipients on a regimen of platelet-inhibiting agents during support and have found that they are likely to have complications of peptic ulcer disease, including upper gastrointestinal bleeding necessitating multiple blood transfusions. About $15 \%$ of our patients in whom bridge to transplantation was attempted had abdominal complications necessitating operative intervention. Severe device-related 


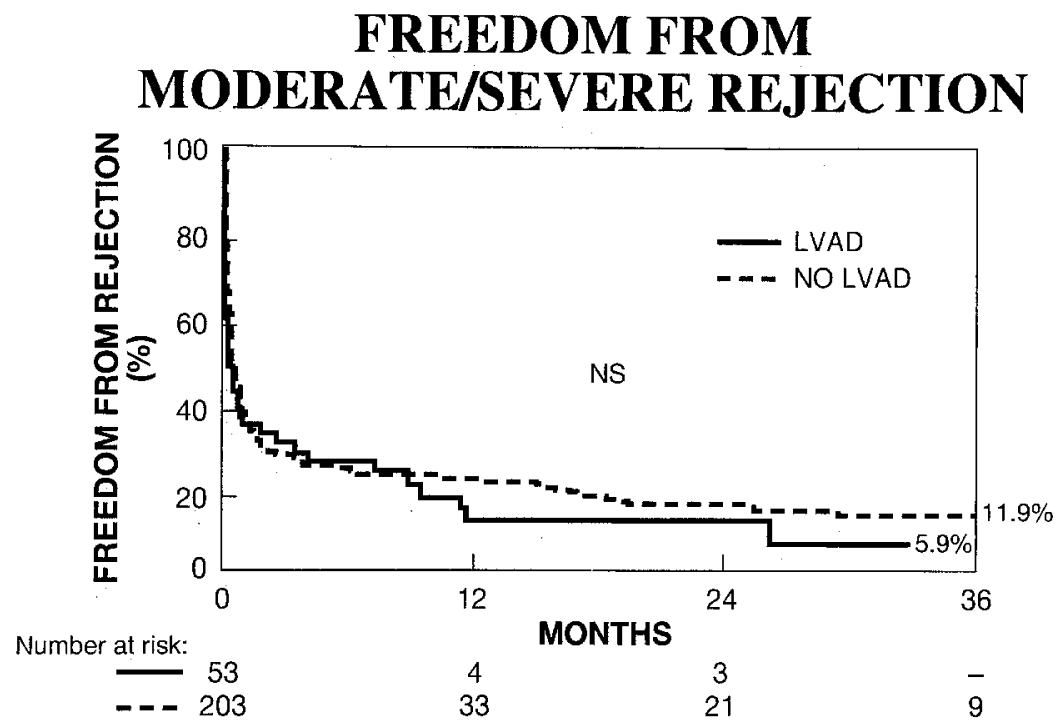

Fig. 2. Actuarial freedom from rejection (\%) of the patients with and without LVAD bridge to transplantation. Deaths were censored.

hemorrhage occurred in an additional $8 \%$, necessitating emergency operative intervention and multiple blood transfusions.

Although LVAD recipients were in cardiogenic shock before bridging, had multiple blood transfusions and infections during support, and underwent complex reoperations, LVAD support did not have a negative influence on the posttransplantation hospital course and did not adversely affect survival after transplantation. Our present experience with the implantable LVAD as a bridge to transplantation agrees with other reports demonstrating that survival of patients who require mechanical circulatory support for bridging is equivalent to that of the cohort of patients not requiring mechanical circulatory support., ${ }^{4,20}$ Our study also shows no significant differences in posttransplantation length of hospital stay and operative mortality between the LVAD and the non-LVAD groups.

Patients in the LVAD group were larger and more likely to have type $\mathrm{O}$ blood group. It has been observed from the UNOS database that the median waiting time for larger patients with type O blood was 319 days in 1993 and that the percentage of deaths on the waiting list was higher in that group. ${ }^{5}$ It is likely that a larger number of these patients will progress to UNOS status I while waiting because of their longer waiting times, and they are therefore more likely to need mechanical support. For these patients, we recommend the use of a portable, battery-powered electric system to facilitate early discharge from the hospital to reduce hospital costs and to improve patient mobility and quality of life during this longer wait. We reported elsewhere ${ }^{19}$ that patients with LVAD bridging are at increased risk for development of anti-HLA antibodies as a result of the multiple blood transfusions they receive during support. In this study, we could not demonstrate any association between HLA sensitization from blood use during LVAD support and the occurrence of vascular and cellular rejection or the number of rejection episodes at one-year of followup. However, the larger number of blood products that LVAD patients received may have contributed to their higher rate of positive $\mathrm{CMV}$ serologic results before transplantation. A larger patient cohort and a longer period of follow-up are required to determine the impact of the extensive blood use in LVAD recipients on the rate of CMV infection after transplantation.

Although the number of patients undergoing transplantation at our institution has not changed significantly during the past 4 years, the frequency of LVAD use has increased remarkably and accounted for one third of the cardiac transplants performed in 1995. Because the competition for scarce organ donors is increasing and LVAD recipients have high priority as status I patients, this trend will probably continue. ${ }^{21}$ The implications for the cost of the cardiac transplantation process are important. The costs of two operations for patients with this bridge (LVAD insertion and transplantation) are higher 
than the costs of transplantation alone for patients who do not need bridging with LVAD support.

Our data summarize a single center's experience with the implantable LVAD as a bridge to transplantation. This study thus avoids the bias seen with multiinstitutional studies, with potentially different patient selection criteria and management before LVAD implantation, during support, and after cardiac transplantation. The study was limited, however, by the fact that the non-LVAD group compared with the LVAD group was neither blinded nor randomized. A drawback of this study is the difference in the mean period of follow up between the two groups. A longer period of follow-up will provide further insight into the incidence of viral and nonviral infections after transplantation, as well as the impact of sensitization during bridging on the long-term incidence of rejection and graft arteriosclerosis.

\section{Conclusion}

LVAD support intensified the donor shortage by keeping alive recipients who otherwise would not have survived to transplantation. Bridging affected transplant demographics, favoring patients who are larger, have ischemic cardiomyopathy, have multiple blood transfusions and complex cardiac operations, are HLA sensitized, and have longer graft ischemic time. Patients with successful bridging wait longer for transplantation than do patients in UNOS status I without bridging but have similar posttransplantation hospital stays and operative mortality rates, with survival also similar to that of patients not requiring LVAD support. The need for LVAD support is not a marker of poor outcome after transplantation, and the impact of bridging on longterm outcomes needs further follow up.

\section{REFERENCES}

1. Bourge RC, Stevenson LA, Naftel DC, et al. Risk factors for death in critically ill patients awaiting cardiac transplantation: a multi-institutional study [abstract]. Circulation 1995; 92(Suppl):I702.

2. Hosenpud JD, Novick RJ, Breen TJ, Keck B, Daily P. The registry of the International Society for Heart and Lung Transplantation: twelfth official report-1995. J Heart Lung Transplant 1995;14:805-15.

3. Portner PM, Oyer PE, Pennington DG. Implantable electric left ventricular assist system: bridge to transplantation and the future. Ann Thorac Surg 1989;47:142-50.

4. Frazier $\mathrm{OH}$, Macris MP, Myers TJ, et al. Improved survival after extended bridge to cardiac transplantation. Ann Thorac Surg 1994;57:1416-22.

5. Frazier OH, Rose EA, McCarthy PM, et al. Improved mortality and rehabilitation of transplant candidates treated with a long-term implantable left ventricular assist system. Ann Surg 1995;222:327-38.

6. Frazier OH, Rose EA, Macmanus Q, et al. Multicenter clinical evaluation of the HeartMate 1000 IP left ventricular assist device. Ann Thorac Surg 1992;53:1080-90.

7. McCarthy PM, Portner PM, Tobler HG, Starnes VA, Ramasamy N, Oyer PE. Clinical experience with the Novacor ventricular assist system: bridge to transplantation and the transition to permanent application. I Thorac Cardiovasc Surg 1991;102:578-87.

8. McCarthy PM, Wang N, Vargo RL. Preperitoneal insertion of the HeartMate 1000 IP implantable left ventricular assist device. Ann Thorac Surg 1994;57:634-8.

9. $\mathrm{Oz} \mathrm{MC,} \mathrm{Goldstein} \mathrm{DJ,} \mathrm{Rose} \mathrm{EA.} \mathrm{Preperitoneal} \mathrm{placement} \mathrm{of}$ ventricular assist devices: an illustrated stepwise approach. J Card Surg 1995;10(4 Pt 1):288-94.

10. Radovancevic B, Frazier $\mathrm{OH}$, Duncan JM. Implantation technique for the HeartMate left ventricular assist device. J Card Surg 1992;7:203-7.

11. Billingham ME, Cary NR, Hammond ME, et al. A working formulation for the standardization of nomenclature in the diagnosis of heart and lung rejection: heart rejection study group. J Heart Transplant 1990;9:587-93.

12. Nakhleh RE, Jones J, Goswitz JJ, Anderson EA, Titus J. Correlation of endomyocardial biopsy findings with autopsy findings in human cardiac allografts. J Heart Lung Transplant 1992;11(3 Pt 1):479-85.

13. Hammond EH, Yowell RL, Nunoda S, et al. Vascular (humoral) rejection in heart transplantation: pathologic observations and clinical implications. J Heart Transplant 1989; 8:430-43.

14. Hammond EH, Hansen JK, Spencer LS, et al. Immunofluorescence of endomyocardial biopsy specimens: methods and interpretation. J Heart Lung Transplant 1993;12: S113-24.

15. Hook S, Caple JF, McMahon JT, Myles JL, Ratliff NB. Comparison of myocardial cell injury in acute cellular rejection versus acute vascular rejection in cyclosporine-treated heart transplants. J Heart Lung Transplant 1995;14:351-8.

16. Ratliff NB, McMahon JT. Activation of intravascular macrophages within myocardial small vessels is a feature of acute vascular rejection in human heart transplants. J Heart Lung Transplant 1995;14:338-45.

17. Lavee J. Influence of PRA and lymphocytotoxic crossmatch on survival after heart transplant. J Heart Lung Transplant 1991;10:921-9.

18. McCarthy PM, Schmitt SK, Vargo RL, Gordon S, Keys TF, Hobbs RE. Implantable LVAD infections: implications for permanent use of the device. Ann Thorac Surg 1996;61:35965.

19. Massad MG, Cook DJ, Vargo R, Gurley S, Schmitt S, McCarthy PM. Factors influencing HLA sensitization in implantable LVAD recipients [abstract]. J Heart Lung Transplant 1996;15:S66.

20. Pennington DG, McBride LR, Miller LW, Swartz MT. Eleven years' experience with the Pierce-Donachy ventricular assist device. J Heart Lung Transplant 1994;13:803-10.

21. Stevenson LW, Warner SL, Hamilton MA, et al. Modeling distribution of donor hearts to maximize early candidate survival. Circulation 1992;86(Suppl 2):II224-30. 


\section{Discussion}

Dr. Eric A. Rose (New York, N.Y.). I congratulate you and your colleagues at the Cleveland Clinic for your excellent results in this challenging patient population with heart failure. It seems clear that this experience is dramatically altering the composition of the transplant recipient pool to include an increasing proportion of device-dependent patients, now exceeding $40 \%$ of those receiving transplants at your institution. You also point out that device use remains an imperfect technology, with persistent problems including anti-HLA sensitization and septicemia, although thromboembolism is now remarkably infrequent.

The increasing success in bridging to transplantation also creates difficult dilemmas in managing the enlarging pool of potential transplant candidates. Bridging to transplantation does not increase the number of transplants performed; rather, it shifts the donor pool away from patients who do not have bridging. You point out that your increasingly frequent use of bridging has intensified the donor shortage at your institution. Furthermore, bridging to transplantation is an expensive process that may not be fully reimbursable for many patients. Regardless of these concerns, increasing experience in extended duration of bridging to transplantation brings us ever closer to using these devices as what may be called "destination therapy."

In our bridge experience with 80 patients at ColumbiaPresbyterian Medical Center, we were able to explant devices because of recovery of left ventricular function in three patients. Two of these three patients survived in short-term follow-up; the third died of rapidly progressive left ventricular failure associated with a viral syndrome 2 months after explantation. We believe that an as yet undefined subset of patients with bridging may spontaneously recover with temporary device support, without the need for transplantation.

I have four questions. First, have you been able to explant any devices from patients without transplantation? Second, can you specifically identify a recipient population without bridging on your waiting list with deterioration or death because of the increasing use of mechanical assistance? Or has improving medical management of your prospective recipient pool overcome this thorny issue? Third, have you compared costs for the patients with bridging with those of both status I and status II patients without bridging in this series? Finally, do you envision the indefinite deferral of transplantation, either because of patient preference or because of increasing competition for donors, in the near future?

Dr. Ricardo Moreno-Cabral (San Diego, Calif.). I also congratulate you for sharing your data with us. We have implanted 16 Thermo Cardiosystems pumps-12 pneumatic and four vented electric-and have performed transplantation in 11 of these patients. Our survival results are quite similar to those presented today.

My questions relate to the subgroup of patients with a high percentage of PRAs. We have a patient whose PRA levels have been greater than $90 \%$ and who has been assisted for now 405 days, probably the longest time a patient has been assisted with a pneumatic Thermo Cardiosystems device. The first question is, have you modified your immunosuppression protocols for this subgroup of patients? The second is, have you noted any evidence of early graft atherosclerosis or decreased 2- to 3-year survival after transplantation among patients with high PRAs or a positive crossmatch result?

Dr. J. Donald Hill (San Francisco, Calif.). I congratulate you and the Cleveland Clinic group on your excellent work. Your conclusions support the other reported experience in bridge to transplantation; that is, the short- and long-term results in LVAD-supported patients are similar to those of patients who only undergo cardiac transplantation, and especially that donor hearts are not wasted. I suggest, however, that in 1996 the question is not whether one can successfully bridge a patient to transplantation with an LVAD in a select group of patients but whether one can bridge all patients who require support?

In your series, 53 patients received LVAD support. How many emergency patients or patients on the transplant waiting list whose condition deteriorated did not qualify for LVAD support for medical reasons (e.g., patient size, right heart failure, arrhythmias, liver failure)? All of these comorbidities/conditions present special problems for LVADs. The question confronting surgeons today is not whether one cannot support carefully selected patients, but how one can support patients who manifest the late signs of congestive heart failure without utilizing biventricular support.

Dr. Cliff Van Meter (New Orleans, La.). I echo the congratulations for your outstanding result in this challenging group of patients both with and without LVAD support. Our experience in New Orleans has been a bit different. In a similar period, we did slightly in excess of 200 transplants. We used LVAD in 12. One did not survive the attempted ventricular support, 10 successfully underwent transplantation and are survivors, and one is receiving ongoing support. In our area, in excess of $85 \%$ of transplants are done in status I patients. We do transplants in very few status II patients. Some questions still remain regarding the optimal timing, not only of LVAD support but also of transplantation after LVAD support. To help in clarifying some of those questions, I have two questions. First, what was the status I patient waiting list mortality in the non-LVAD group? Second, excluding status II patients, was there a significant difference between posttransplantation hospital stays of the status I LVAD group versus the non-LVAD patient population? More specifically, we have found a remarkably shorter posttransplantation hospital stay among those with LVAD support versus those without.

Dr. Massad. I thank the discussants for their comments. To answer Dr. Rose's first question, we have not been able to explant any devices from patients without transplantation as a result of recovery of their native myocardium. In response to your second question, although it is hard for me to recall a status I transplant candidate without bridging who had deteriorated condition or died merely because of lack of a donor as a result of the use of mechanical assistance, I concur that the improvement in the medical management of our prospective transplant recipient pool has played a major role in overcoming this problem. In addition to that, and to accommodate the group of LVAD recipients, we have expanded our donor 
selection criteria to include those who were considered "marginal donors," without a demonstrably negative impact of the use of those so-called "marginal donors" on survival. I do recall that we had six status II patients whose symptoms progressed and required LVAD support in our transplant group. With respect to cost, I believe at our institution the median cost of a bridged transplant is almost twice that of a nonbridged transplant. I am certain that cost is going to be a significant issue in the near future. I envision the indefinite deferral of transplantation with future improvements in circulatory assistance technology.

To answer Dr. Moreno-Cabral's questions, we had few patients who had high PRA levels during LVAD support and were able to deal with them on individual basis. We do not routinely perform prospective crossmatching on all our transplant candidates. With respect to the highly sensitized LVAD recipients, we assume that they are going to be seropositive and deal with that accordingly. We subjected four such patients (mean PRA 97\%) to plasmapheresis during support when they were high on the waiting list to provide a window for them to undergo transplantation; with that, we were able to drop their quantitative antibody levels by $77 \%$, as measured by flow cytometric analysis. We perform retrospective crossmatching on all our patients after transplantation and have modified the immunosuppression protocol in the highly sensitized group to include induction with monoclonal antibodies (OKT3) for 2 weeks and continued plasmapheresis after transplantation for 3 to 4 weeks. We have also added cyclophosphamide or mycophenalate mofetil instead of azathioprine. From the data that I have presented, early graft arteriosclerosis and actuarial survival estimates in the overall sensitized LVAD recipients and the nonbridged transplant recipients were similar.

I echo the concerns of Dr. Hill with respect to the patients manifesting the late signs of congestive heart failure, who form a more challenging group. However, in most of our LVAD-bridged patients we were able to reverse the state of right heart failure with inotropes and pulmonary vasodilators. Right heart failure necessitated temporary implantation of a right ventricular-assist device in only $12 \%$ of our transplant recipients. In this subset of patients, the need for a right ventricular-assist device was a marker of poor outcome; it was associated with a significantly lower transplantation rate. Approximately one of every eight deaths among our LVAD recipients was a result of right ventricular failure necessitating right ventricular-assist device support.

To answer the questions that Dr. Van Meter raised, the mortality for the UNOS status I listed patients without bridging while waiting was not different from that of LVAD recipients. The mean posttransplantation hospital stays of both groups were also similar. 